\title{
An Evaluation of Sources of Erosion in Hall Thrusters
}

\author{
John T. Yim, Michael Keidarł ${ }^{\dagger}$ and Iain D. Boyd ${ }^{\ddagger}$ \\ University of Michigan, Ann Arbor, MI 48109
}

\begin{abstract}
As missions involving Hall thrusters become more extended and further reaching, lifetime issues arise in which the thrusters begin to need to have lifespans of 10000 hours and upwards. The main limiting factor and failure mode of stationary plasma thrusters (SPT) is the erosion of the acceleration channel walls. The walls erode from a sputtering process caused by ions impacting the surfaces. This work investigates two sources of the ion flux to the walls causing erosion. In a previous study, scattering collisions were examined to observe their contribution in deflecting ions to the walls. Here we use a more sophisticated hydrodynamic model of the plasma to determine the ion flux to the walls as caused by the plasma dynamics as well as including near-wall interactions. Both models provide results that match well with experimental data. However, the scattering collisions model makes a number of debatable assumptions. The hydrodynamic model shows that the near-wall effects have a significant role in the erosion process.
\end{abstract}

\section{Nomenclature}

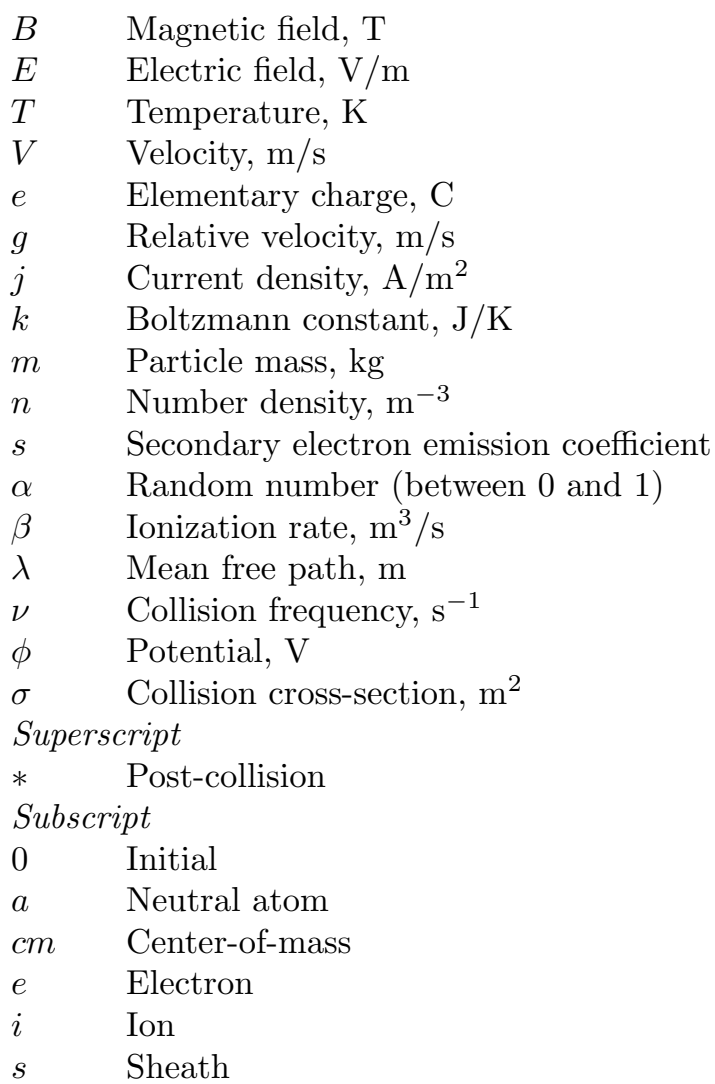

${ }^{*}$ Graduate student, Department of Aerospace Engineering, AIAA Student Member.

${ }^{\dagger}$ Assistant Research Scientist, Department of Aerospace Engineering, AIAA Senior Member.

¥Professor, Department of Aerospace Engineering, AIAA Associate Fellow. 


\section{Introduction}

Hall thrusters, like other forms of electric propulsion, provide an efficient means of space propulsion. However, the high specific impulse comes at a cost of reduced thrust. Transit time of spacecraft using these devices is thus increased in comparison to chemical propulsion systems, as is the duration of operation for these devices. As the mission envelope is expanded for Hall thrusters beyond Earth orbit, higher power, higher specific impulse, higher efficiency, and longer lifetimes are desired. ${ }^{1}$ Experimental validation of thruster lifetimes requires much time and high cost. An accurate computational simulation of Hall thruster erosion would provide an efficient and effective tool to aid in the design and validation process alongside experimental means.

The main life-limiting factor for a stationary plasma thruster (SPT)-type Hall thruster is the erosion of the acceleration channel walls. ${ }^{2}$ This erosion by sputtering of wall material is caused by ions impinging onto the discharge chamber surfaces. There have been several previous attempts at establishing a model for the erosion of the Hall thruster channel walls. ${ }^{2-5}$ However, they are mostly semi-empirical in nature. The next step is to develop a more physics-based approach to erosion modeling.

In previous work, a time-dependent erosion model was developed based on ion scattering collisions within the thruster. ${ }^{6}$ This work hypothesized that the sputtering of the wall material is caused mainly by ions scattered from the main plasma flow to the walls. The reasoning behind this is derived from the measured profile of the erosion rate versus time obtained for the SPT-100 thruster, shown in Fig. $1 .{ }^{7}$ As can be seen, the erosion rate drops relatively rapidly early on, but then tapers off later. Possible explanations for these trends have ranged from a two-mechanism erosion process to a logarithmic dependence. ${ }^{3,5}$

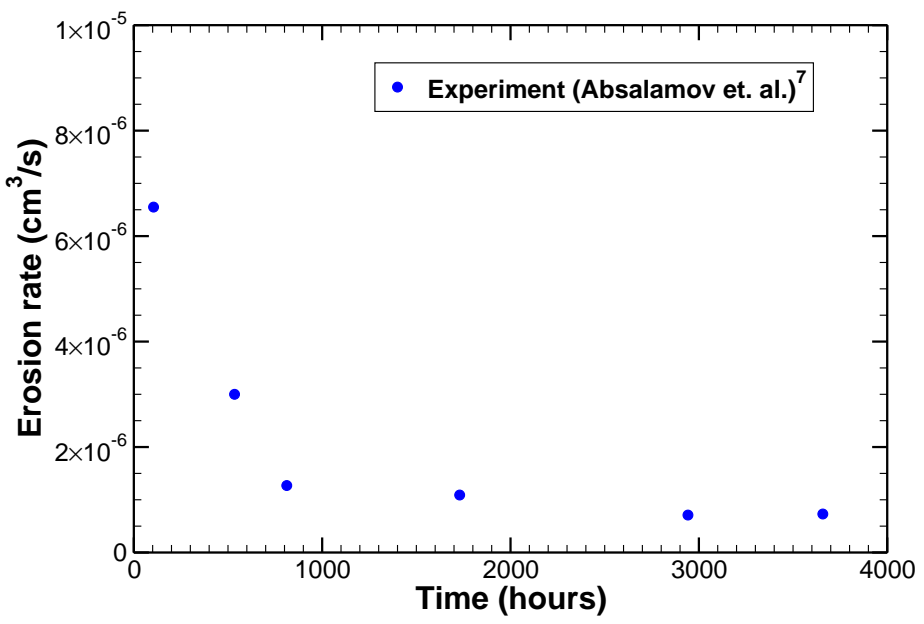

Figure 1. The experimentally measured total volumetric erosion rate as a function of thruster operation time.

A physical process behind a logarithmic profile could be explained by scattering collisions. As the walls erode away, the distance for an ion to reach the surface increases. Then the probability that an ion reaches the walls before being scattered again is reduced. From kinetic theory, the number of ions reaching the walls traveling through the eroded distance, $l$, is given by

$$
n=n_{0} \exp (-l / \lambda)
$$

which provides a logarithmic influence on the erosion rate profile over time. This model, however, in spite of matching relatively well with experimental results, makes several assumptions that can be improved upon. Thus there is the motivation to develop a model that incorporates more of the physics of the plasma flow while providing usable erosion estimates and predictions.

The scattering collisions model and a hydrodynamic model are described in more detail in the following section. The sputtering model used in determining the erosion rates is also described in the next section. 
The results of the two models determining the ion flux are then shown and compared to each other. Finally, conclusions and suggestions for future work are discussed in the final section.

\section{Models}

The erosion rates along the thruster channel walls are calculated by determining the ion flux to the walls and the subsequent sputtering that occurs. The sputtering model is the same for both the scattering collisions model as well as the hydrodynamic model. The primary difference between the two models lies in how the ion fluxes and impingement characteristics at the walls are calculated.

\section{A. Ion flux models}

\section{Scattering collisions}

For the scattering collisions model, a one-dimensional formulation is employed. Through a simplification of the electron momentum equation, the axial electric field is assumed to be proportional to the radial magnetic field strength. This assumes the electron drift velocity is independent of the magnetic field, which is debatable. From the derived electric field along with conservation of mass, profiles of the average ion velocity and number density along the channel length can be calculated.

Kinetic theory is then applied to obtain the collision properties. A cross section measured for xenon collisions between singly charged ions and neutral atoms is used. ${ }^{8}$ Using the cross section, the collision rate can be calculated from

$$
Z_{i a}=n n_{a}\left\langle\sigma_{i a} g\right\rangle
$$

From the collision rate, the ion flux diverted from the main flow to the walls is found. This ion flux is adjusted by the secondary scattering away from the walls through Eq.(1).

An isotropic scattering process is assumed and the post-collision velocity vector components can then be subsequently found. The axial and radial components are randomly determined through

$$
\begin{gathered}
V_{z}^{*}=V_{c m_{z}}+\frac{m_{a}}{m_{i}+m_{a}}|g| \cdot \alpha_{1} \\
V_{r}^{*}=V_{c m_{r}}+\frac{m_{a}}{m_{i}+m_{a}}|g| \cdot \sqrt{1-\alpha_{1}} \cdot \cos \left(\alpha_{2} \cdot 2 \pi\right)
\end{gathered}
$$

The post-collision values are used only to calculate the incident angles with which the ions impact the walls. The energies of the post-scattered ions are approximated by the pre-collision energies. This approach is used to incorporate the fact that the ions undergo further electrostatic acceleration while traveling to the wall. The axial location of the ion collisions with the walls is assumed to be the same location as they scattered in.

The wall impact parameters are then used in the sputtering model to determine the erosion rates along the wall at a particular time. The wall is eroded away accordingly for each time-step and the geometry updated. Then new values for each of the properties are calculated to determine the new erosion rates. More complete details for this model are provided by Manzella et al. ${ }^{6}$

\section{Hydrodynamic}

The bulk of the hydrodynamic model is based on the work by Keidar et al. where the ion mass and momentum conservation equations in an axially symmetric framework are solved iteratively to reach a steady-state solution. ${ }^{9}$

$$
\begin{gathered}
\nabla \cdot\left(n \mathbf{V}_{i}\right)=\beta n n_{a} \\
n m_{i}\left(\mathbf{V}_{i} \nabla\right) \mathbf{V}_{i}=e n \mathbf{E}-\beta n m_{i} n_{a} \mathbf{V}_{a}
\end{gathered}
$$

Here the source term in the continuity equation is from ionization of the neutrals. In the momentum equation, the ions are considered to be unmagnetized and cold. These are reasonable assumptions since the ion gyro radius is much greater than the channel length and the ion temperature is negligible compared to the electron temperature. ${ }^{10}$ Neutrals are considered to be in a one-dimensional flow with a steady velocity. The neutral number density is calculated from a mass conservation equation similar to Eq.(5). 
In addition to the ion equations, the electron momentum and energy equations are solved. The momentum equation is solved both along and across the magnetic field. The electron system is assumed to be non-inertial.

$$
0=-e n(\mathbf{E}+\mathbf{V} \times \mathbf{B})-\nabla\left(n k T_{e}\right)-n m_{e} \nu_{\mathrm{eff}} \mathbf{V}_{e}
$$

The effective electron collision frequency, $\nu_{\text {eff }}$, is modeled as a combination of the collisions of electrons with ions, neutrals, the walls, as well as including anomalous transport, or Bohm diffusion. For this model, only the radial component of the magnetic field is considered and the electron temperature is assumed to be constant along the magnetic field lines. From these assumptions, a thermalized potential can be used to simplify the calculation of the electric field.

$$
\phi-\frac{k T_{e}}{e} \ln n=\mathrm{const}
$$

The electron energy equation used is

$$
\frac{3}{2} \frac{\partial\left(j_{e} T_{e}\right)}{\partial z}=Q_{\text {joule }}-Q_{\text {ion }}-Q_{\text {wall }}
$$

where $Q_{\text {joule }}$ is the heat addition due to Joule heating, $Q_{\text {ion }}$ is the energy lost due to ionization, and $Q_{\text {wall }}$ is the energy lost to the walls.

The plasma-wall interaction within Hall thrusters is an area that is still not perfectly understood and is under investigation. ${ }^{11,12}$ The hydrodynamic model presented here incorporates the effects of the plasma sheath into the calculations. The lateral boundaries of the computational domain are considered to be at the sheath-presheath interface, and the appropriate boundary conditions are applied. Instead of assuming the Bohm condition at the sheath edge, a smooth presheath-sheath patching technique is applied where the radial velocity at the sheath entrance and electric field are determined as a part of the solution. ${ }^{13-15}$ Thus in the calculation of the electron wall losses ${ }^{9}$

$$
Q_{\mathrm{wall}}=\nu_{\mathrm{wall}} n k T_{e}\left(2+(1-s) \ln \phi_{s}\right)
$$

the potential drop across the sheath, $\phi_{s}$ is given by

$$
\phi_{s}=\frac{1-s}{V_{s} \sqrt{2 \pi m_{e} / k T_{e}}}
$$

where $V_{s}$ is the ion radial velocity at the entrance of the sheath, which is not necessarily the Bohm velocity. The effects of secondary electron emission are also included, as seen in the equations above. The secondary electron emission coefficient, $s$, is chosen to vary with the electron temperature linearly as presented by Dunaevsky et al. for boron nitride. ${ }^{16}$

$$
s \approx 0.54+(1-0.54) \frac{T_{e}(\mathrm{eV})}{40}
$$

It should be mentioned that Eq.(11) is valid before the charge-saturation regime is reached. This occurs when the secondary electron emission coefficient is below a critical value ${ }^{17}$

$$
s_{\text {crit }}=1-8.3 \sqrt{\frac{m_{e}}{m_{i}}}=0.983(\text { for Xe) }
$$

From the calculation of the properties at the sheath-presheath interface, the ion flux to the walls is determined. Since the wall will have a negative potential with respect to the bulk plasma as long as the secondary electron emission coefficient is below unity, the ions will also experience acceleration across the sheath with an energy on the order of the electron temperature. This effect is included in the determination of the impact parameters that are used in the sputtering model. The ions are considered to impact the walls as a monoenergetic stream since it is assumed that they are cold.

The hydrodynamic code is run iteratively on a regular structured mesh until it converges to a steady state solution. It is realized that a true steady state condition is never reached in Hall thrusters due to the presence of many low and high frequency oscillatory effects in the plasma. ${ }^{18}$ However, since the erosion process is much slower than the time scale of these oscillations, obtaining a solution which represents the average properties is sufficient for use in determining the sputtering and erosion. Due to the nature of the regular structured mesh this model uses, however, it is difficult to incorporate the changing geometry of the thruster as it erodes away. Thus, only the beginning-of-life (BOL) erosion properties are evaluated with this model for now. Future work will include the geometry changes and their effect on the erosion rate over time. 


\section{B. Sputtering model}

Experiments and simulations show that sputtering is a function of four main parameters: wall material, ion species, and the incident angle and energy of the impinging ion. ${ }^{4,19-22}$ For our purposes here, we use the data obtained for xenon ions bombarding boron nitride. ${ }^{19}$ Curve fits applied to the sputtering dependence on both incident angle and energy are shown in Figs. 2 and 3. The angular sputtering dependence is normalized so that when multiplied by the energy dependence, the sputter yield based on both incident angle and energy is obtained. It should be noted that low energy $(<100 \mathrm{eV})$ sputter data is scarce and difficult to obtain for insulators such as boron nitride. However, this region is of importance for applications of Hall thruster wall material sputtering since ion energies will typically be less than that of the discharge voltage of the thruster, which in this case is $300 \mathrm{~V}$. For the results here, we have assumed a $50 \mathrm{eV}$ threshold for sputtering and applied a logarithmic fit to the energy dependence data.

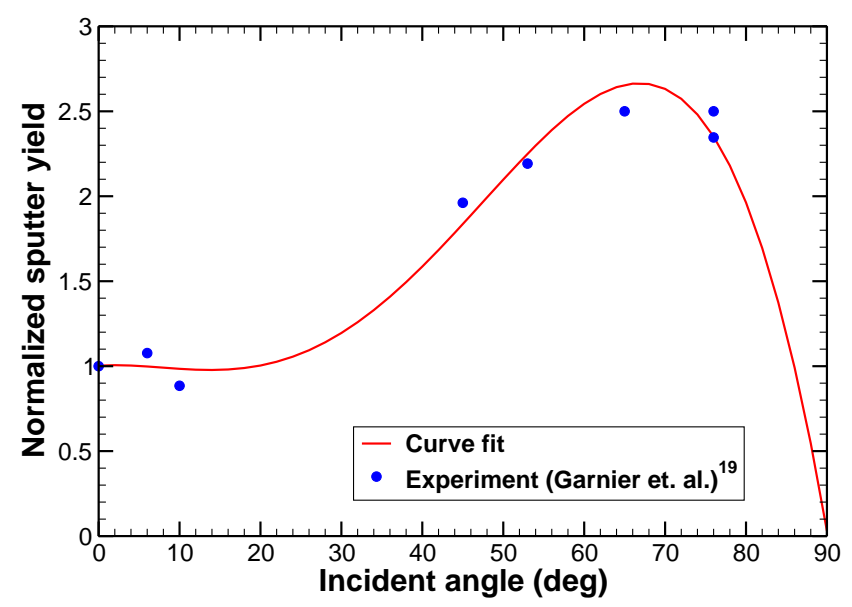

Figure 2. The normalized angular dependence of the sputter yield.

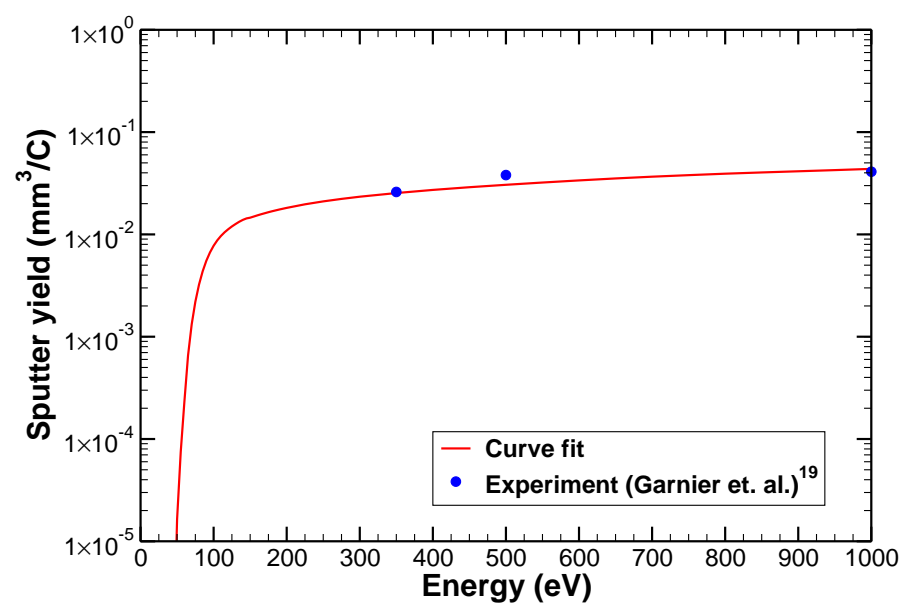

Figure 3. The energy dependence of the sputter yield at normal incidence 


\section{Results}

For both of the models, the same SPT-100 thruster geometry and operating conditions are used as shown in Table 1. The same profile of the radial magnetic field strength is also used for both models. For the hydrodynamic model, a mesh of 2400 by 314 cells is used for 20 iterations. The total run time for the hydrodynamic model is on the order of a minute, comparable to that of the scattering collisions model. The profiles of the plasma potential for both of the models are shown in Fig. 4. The hydrodynamic model shows a greater drop within the channel by more than $100 \mathrm{~V}$ over the scattering collisions model. This discrepancy stems from the way the electric field is calculated in the two models. The scattering collisions model bases the electric field strength as proportional to the magnetic field whereas the hydrodynamic model calculates it from the electron momentum equation. A contour plot of the plasma density within the channel calculated by the hydrodynamic model is shown in Fig. 5. In comparison, the number density of ions as modeled by the one dimensional scattering collisions model monotonically decreases from a peak value of around $2.4 \times 10^{18} \mathrm{~m}^{-3}$ near the anode. ${ }^{6}$ Figure 6 displays the electron temperature along the channel obtained from the hydrodynamic model. The shape of the profile, where the peak temperature is at the exit, is due to the fact that the magnetic field profile used in this model also has its peak at the exit. The scattering collisions model does not make use of the electron temperature.

\begin{aligned} & \hline \hline Parameter Value \\ & \hline outer diameter: $0.1000 \mathrm{~m} \\ &$ inner diameter: $0.0686 \mathrm{~m} \\ &$ channel length: $0.024 \mathrm{~m} \\ &$ mass flow rate: $4.9 \times 10^{-6} \mathrm{~kg} / \mathrm{s} \\ &$ discharge voltage: $300 \mathrm{~V} \\ &$ discharge current: $4.5 \mathrm{~A} \\ &$\hline \hline\end{aligned}

Table 1. Thruster operating conditions

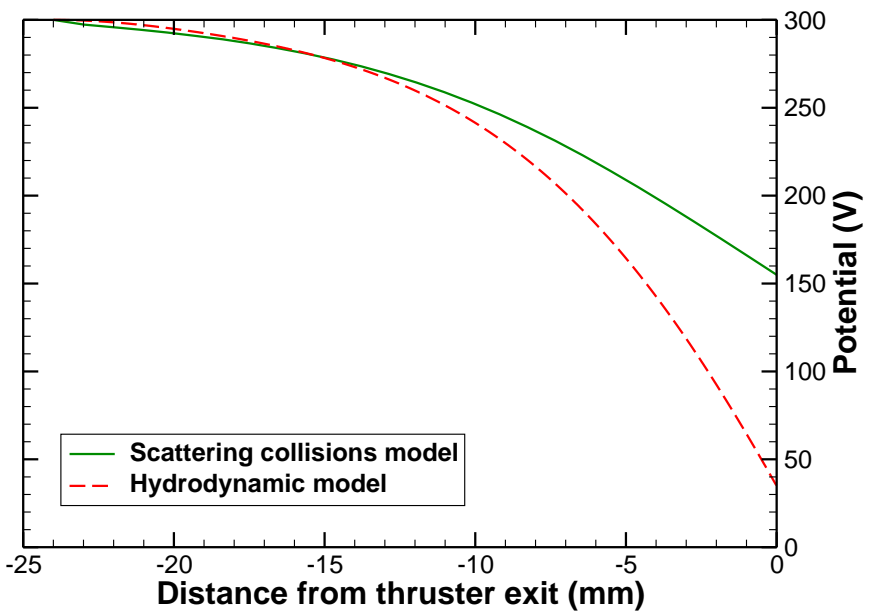

Figure 4. Potential profiles within the thruster. 


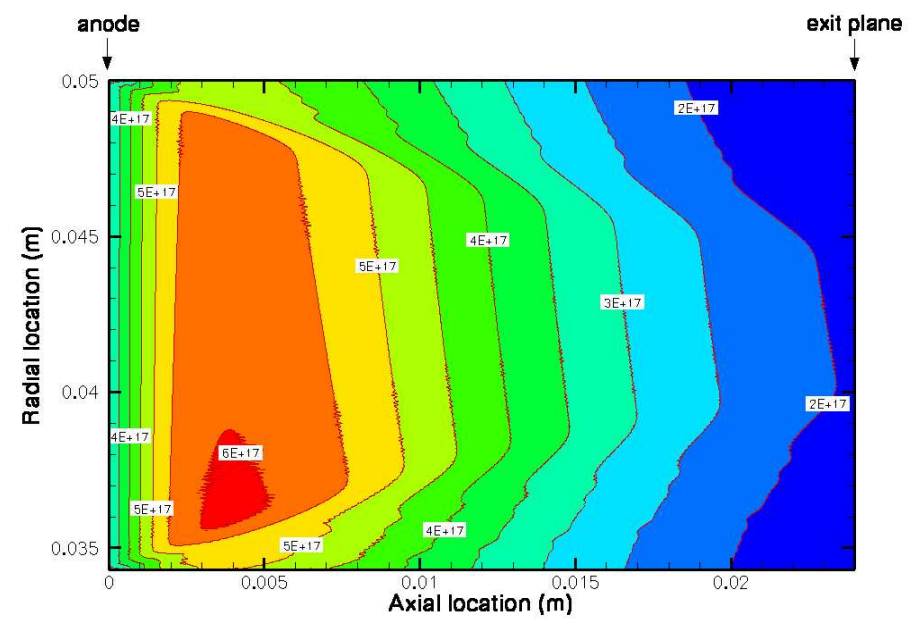

Figure 5. The plasma density $\left(\mathrm{m}^{-3}\right)$ within the thruster calculated by the hydrodynamic model.

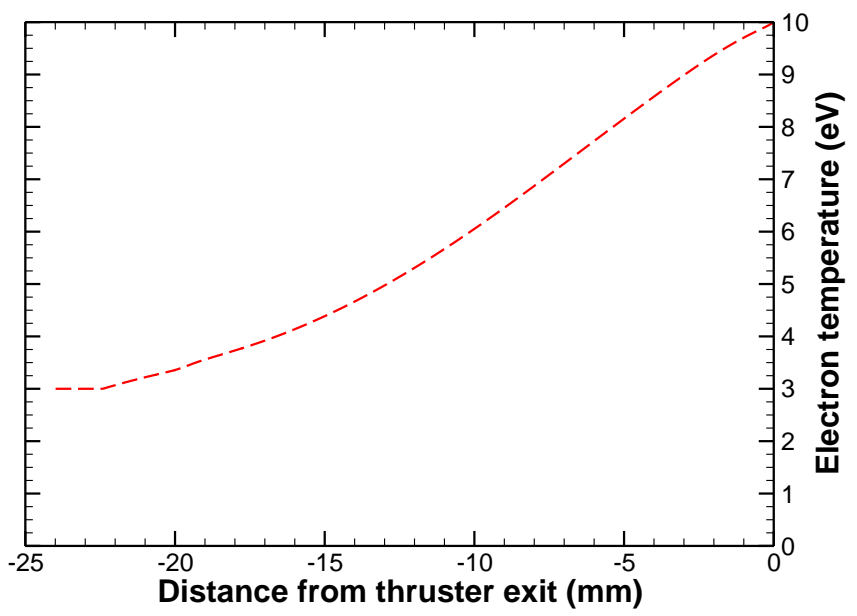

Figure 6. The electron temperature along the channel calculated by the hydrodynamic model. 
As mentioned earlier, the results from the scattering collisions model match surprisingly well with the experimentally measured data. Figure 7 shows sample erosion profiles after specified hours of operation. The lines show the erosion profiles found by the scattering collisions model while the points represent the experimental data. These results were only obtained, however, after increasing the neutral atom number density by a factor of two.
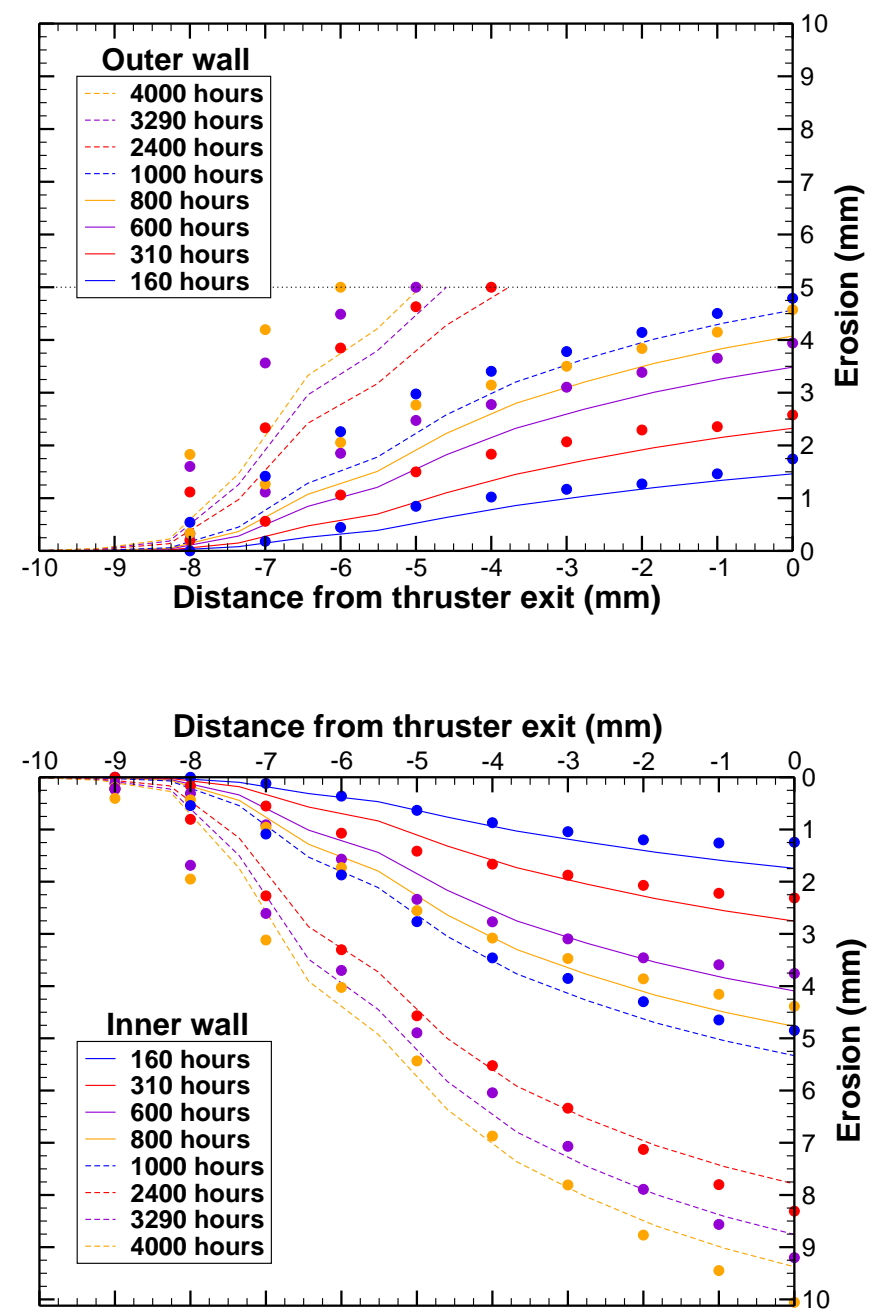

Figure 7. Erosion profiles along the outer and inner walls calculated by the scattering collisions model along with experimental measurements.

Since the hydrodynamic model currently is not able to adapt to changing geometries as the thruster erodes away, only BOL data is calculated. Instead of showing the erosion profiles, the erosion rate profiles along the outer wall of the channel are shown in Fig. 8. The inner wall erosion rate profiles are similar, though they are a little lower. Erosion distances cannot be directly obtained from this data; extrapolation from the initial profile is difficult at the BOL conditions since the erosion rate is the highest at this point as well as having the steepest gradient.

The sensitivity of the results to the acceleration across the sheath is explored. As stated earlier, the ions experience an acceleration across the sheath due to the potential drop. The potential drop as described by Eq.(11) is used. Potential drops of $1 / 2 T_{e}, T_{e}$, and $2 T_{e}$ are also evaluated since the space charge saturation 


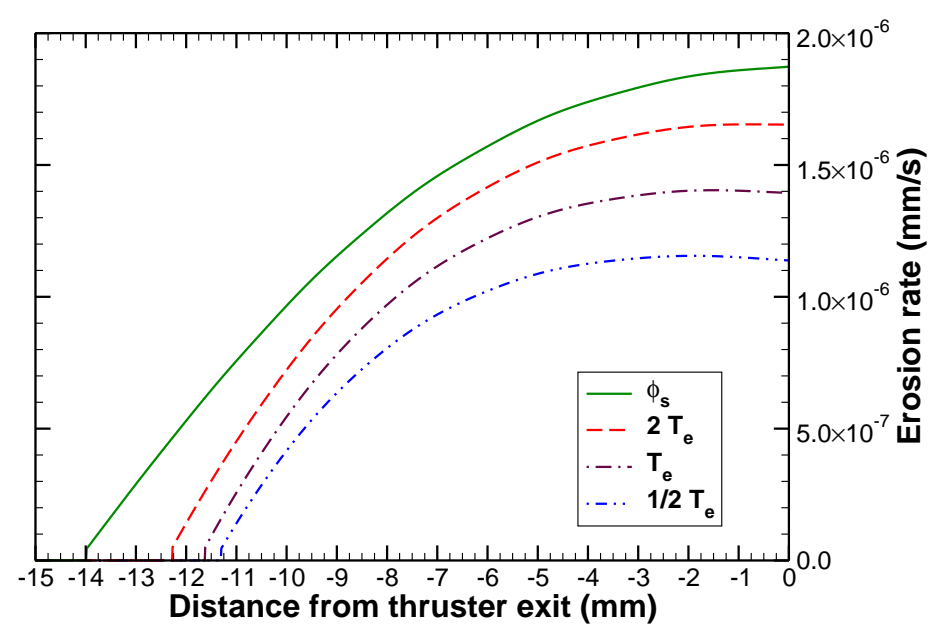

Figure 8. The profiles of the erosion rate along the outer wall as calculated by the hydrodynamic model.

limit may be reached where the voltage drop would deviate from Eq.(11). These values aid in displaying the trends of the effect the potential drop has on the erosion rates. As expected, the increase in the potential drop results in increased erosion rates since the ions are striking the wall surfaces with a greater energy.

One main qualitative difference between the two models seen here is the location where erosion begins. Whereas experimental data and the scattering collisions model show erosion does not occur upstream of 10 $\mathrm{mm}$ from the thruster exit, the hydrodynamic model shows erosion beginning more than $11 \mathrm{~mm}$ from the exit plane. The one millimeter difference can be explained by the potential profiles shown in Fig. 4 . The hydrodynamic modeled potential drops $50 \mathrm{~V}$, the sputtering threshold value, about a millimeter upstream of where the scattering collisions model calculates it. The other upstream values stem directly from the fact that the modeled sheath potential drops are higher, thus pushing the ion impact energies past the threshold value earlier.

For a more direct comparison between the two models, the total volumetric erosion rate, as summed over the entire channel, is plotted in Fig. 9. Here the logarithmic dependence of the erosion rate over time is clearly shown by the scattering collisions model. The experimental data is represented fairly well by the logarithmic decrease. Since the acceleration across the sheath directly influences the ion impact configurations, the results show some variance in the total erosion rate as seen in Fig. 9 based on the sheath potential drop. The results are both higher and lower than the BOL total volumetric erosion rate suggested by the experimental results, but overall are within the same order of magnitude. Again, at this stage the hydrodynamic model only provides results at the BOL conditions.

\section{Conclusion}

In this study, two different approaches (the scattering and hydrodynamic models) were employed to model ion flux to the walls of a Hall thruster. Combining these models with a sputtering model allowed calculation of wall erosion rates. Even though the scattering model provides fairly accurate results in comparison to the experimental data, there is room for improvement particularly in reducing the number of assumptions needed to achieve those results. These assumptions include independence of the electron drift velocity on the magnetic field, ions impacting the walls in the same axial location they were scattered in, and a doubling of the neutral number density. An axisymmetric hydrodynamic model was used to more accurately depict the nature of the plasma flow within the acceleration channel of a Hall thruster. In addition to implicitly including some of the effects of particle kinetics, the hydrodynamic model is based on the natural ion flux to the walls that are not the direct result of particle collisions. 


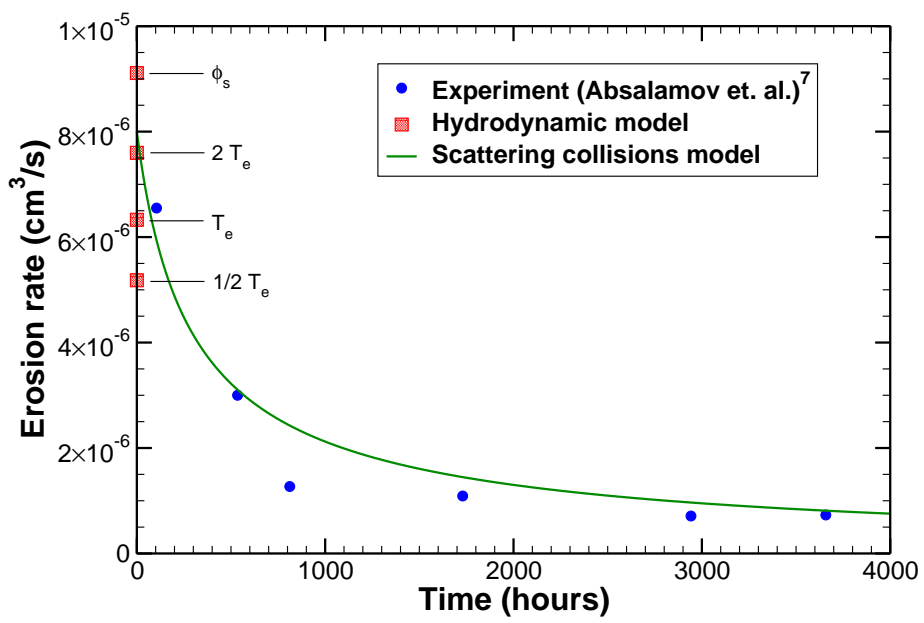

Figure 9. The total erosion rate

Plasma-wall dynamics were included. The scattering collisions model basically ignores plasma-wall effects. With the hydrodynamic model, the plasma-sheath boundary was used to determine the ion flux to the walls. The acceleration of ions across the sheath was also included. The sensitivity of the results to the sheath potential drop was quite large. However, the results, at BOL at least, are promising as they were located within an order of magnitude of what the experimental data suggest.

The next step is to adjust the hydrodynamic code such that it can handle changing geometries and determine the erosion rates over time as the channel walls degrade. This will probably require using an unstructured adaptive mesh rather than the regular structured mesh currently used. Hopefully when the hydrodynamic model is further developed, it can provide more insight into the processes that contribute to the profile of the erosion rate over time. It might be due to purely geometrical reasons, or it might involve more complicated processes in the near-wall region. As the channel erodes away, the wall angles will change and perhaps that of the main plasma flow as well, the densities will be affected, and the boundary conditions will have to adjust among other effects. Ultimately, it is hoped that further knowledge of the factors which contribute to erosion can be used to aid the design and development of future thrusters to reduce erosion and increase lifetimes.

Of course, there are other factors to be considered as development of the hydrodynamic code continues. The effects of multiply charged ions are ignored for now, though they will affect the erosion rates due to their higher energies even though their fractions are low. The electron energy balance can be improved by investigating additional terms such as thermal conduction. The near-wall processes are still not well understood and deserve further consideration; sheath conditions and near-wall conductivity are a few areas that can be continued to work on. Curvature of the magnetic fields also will affect the plasma flow, especially in the areas near the walls where the curvature is generally more pronounced. The sputtering model must also be improved, especially in the low-energy regions. There is also speculation on the roughness of the surface, which would affect the angular dependence significantly. In spite of the many areas listed here that require future work, the results given in this paper from the hydrodynamic plasma model show that the ion flux to the walls causing erosion can be adequately described by the natural plasma dynamics within the thruster channel without using overly reaching assumptions.

\section{Acknowledgments}

Part of this work was supported by NASA Glenn Research Center. The first author would also like to acknowledge support from NSF through a Graduate Research Fellowship. 


\section{References}

${ }^{1}$ Jacobson, D. T., Manzella, D. H., Hofer, R. R., and Peterson, P. Y., "NASA's 2004 Hall Thruster Program," 40th AIAA/SAE/ASME/ASEE Joint Propulsion Conference and Exhibit, 2004, AIAA 2003-3600.

${ }^{2}$ Clauss, C., Day, M., Kim, V., Kondakov, Y., and Randolph, T., "Preliminary Study of Possibility to Ensure Large Enough Lifetime of SPT Operating Under Increased Powers," 33rd AIAA/SAE/ASME/ASEE Joint Propulsion Conference and Exhibit, 1997, AIAA 97-2789.

${ }^{3}$ Maslennikov, N. A., "Lifetime of the Stationary Plasma Thruster," 24th International Electric Propulsion Conference, 1995, IEPC 95-75.

${ }^{4}$ Abgaryan, V. et al., "Calculation Analysis of the Erosion of the Discharge Chamber Walls and Their Contamination During Prolonged SPT Operation," 30th AIAA/SAE/ASME/ASEE Joint Propulsion Conference and Exhibit, 1994, AIAA 94-2859.

${ }^{5}$ Baranov, V., Nazarenko, Y., and Petrosov, V., "The Wear of the Channel Walls in Hall Thrusters," 27th International Electric Propulsion Conference, 2001, IEPC 01-48.

${ }^{6}$ Manzella, D. H., Yim, J. T., and Boyd, I. D., "Predicting Hall Thruster Operational Lifetime," 40th AIAA/SAE/ASME/ASEE Joint Propulsion Conference and Exhibit, 2004, AIAA 2004-3953.

${ }^{7}$ Absalamov, S. K. et al., "Measurement of Plasma Parameters in the Stationary Plasma Thruster (SPT-100) Plume and Its Effect on Spacecraft Components," 28th AIAA/SAE/ASME/ASEE Joint Propulsion Conference and Exhibit, 1992, AIAA 92-3156.

${ }^{8}$ Boyd, I. D. and Dressler, R. A., "Far-field Modeling of the Plasma Plume of a Hall Thruster," Journal of Applied Physics, Vol. 92, Aug. 2002, pp. 1764-1774.

${ }^{9}$ Keidar, M., Boyd, I. D., and Beilis, I. I., "Plasma Flow and Plasma-Wall Transition in Hall Thruster Channel," Physics of Plasmas, Vol. 8, No. 12, Dec. 2001, pp. 5315-5322.

${ }^{10}$ Ahedo, E., Gallardo, J. M., and Martinez-Sanchez, M., "Model of the Plasma Discharge in a Hall Thruster with Heat Conduction," Physics of Plasmas, Vol. 9, No. 9, Sept. 2002, pp. 4061-4070.

${ }^{11}$ Ahedo, E., Martinez-Cerezo, P., and Martinez-Sanchez, M., "Model of Plasma-Wall Interaction Effects in a Hall Thruster," 37th AIAA/SAE/ASME/ASEE Joint Propulsion Conference and Exhibit, 2001, AIAA 2001-3323.

${ }^{12}$ Roy, S. and Pandey, B. P., "Modeling the Effect of Plasma-Wall Interaction in a Hall Thruster," 41st Aerospace Sciences Meeting and Exhibit, 2003, AIAA 2003-493.

${ }^{13}$ Godyak, V. A. and Sternberg, N., "Smooth Plasma-Sheath Transition in a Hydrodynamic Model," IEEE Transactions on Plasma Science, Vol. 18, No. 1, Feb. 1990, pp. 159-168.

${ }^{14}$ Beilis, I. I., Keidar, M., and Goldsmith, S., "Plasma-Wall Transition: The Influence of the Electron to Ion Current Ratio on the Magnetic Presheath Structure," Physics of Plasmas, Vol. 4, No. 10, Oct. 1997, pp. 3461-3468.

${ }^{15}$ Beilis, I. I. and Keidar, M., "Sheath and Presheath Structure in the Plasma-Wall Transition Layer in an Oblique Magnetic Field," Physics of Plasmas, Vol. 5, No. 5, May 1998, pp. 1545-1553.

${ }^{16}$ Dunaevsky, A., Raitses, Y., and Fisch, N. J., "Secondary Electron Emission from Dielectric Materials of a Hall Thruster with Segmented Electrodes," Physics of Plasmas, Vol. 10, No. 6, June 2003, pp. 2574-2577.

${ }^{17}$ Hobbs, G. D. and Wesson, J. A., "Heat Flow Through a Langmuir Sheath in the Presence of Electron Emission," Plasma Physics, Vol. 9, 1967, pp. 85-87.

${ }^{18}$ Choueiri, E. Y., "Plasma Oscillations in Hall Thrusters," Physics of Plasmas, Vol. 8, No. 4, April 2001, pp. 1411-1426.

${ }^{19}$ Garnier, Y., Viel, V., Roussel, J.-F., and Bernard, J., "Low-energy Xenon Ion Sputtering of Ceramics Investigated for Stationary Plasma Thrusters," Journal of Vacuum Science and Technology A, Vol. 17, No. 6, Nov/Dec 1999, pp. 3246-3254.

${ }^{20}$ Kim, V., Kozlov, V., Semenov, A., and Shkarban, I., "Investigation of the Boron Nitride Based Ceramics Sputtering Yield Under Its Bombardment by Xe and Kr Ions," 27th International Electric Propulsion Conference, 2001, IEPC 01-073.

${ }^{21}$ Chen, M., Rohrbach, G., Neuffer, A., Barth, K.-L., and Lunk, A., "Simulation of Boron Nitride Sputtering Process and Its Comparison with Experimental Data," IEEE Transactions on Plasma Science, Vol. 26, No. 6, Dec. 1998, pp. $1713-1717$.

${ }^{22}$ Promokhov, A. A., Mosunov, A. S., Elovikov, S. S., and Yurasova, V. E., "Features of Sputtering of Nitrides with Various Component Mass Ratios," Vacuum, Vol. 56, 2000, pp. 247-252. 